\title{
Image View
}

National Cancer Institute

\section{Source}

National Cancer Institute. Image View. NCI Thesaurus. Code C69203.

An indication of the orientation of the image plane. 